\title{
Cytochrome P450-derived eicosanoids and vascular dysfunction in coronary artery disease patients
}

\author{
Robert N. Schuck ${ }^{a}$, Katherine N. Theken ${ }^{a}$, Matthew L. Edin ${ }^{e}$, Melissa Caughey ${ }^{b, d}$, Almasa \\ Bass $^{a}$, Kyle Ellis ${ }^{a}$, Bryant Tran ${ }^{a}$, Savanna Steele ${ }^{a}$, Brian P. Simmons ${ }^{a}$, Fred B. Lih ${ }^{e}$, \\ Kenneth B. Tomer ${ }^{\mathrm{e}}$, Michael C. Wuc ${ }^{\mathrm{c}}$, Alan L. Hinderliter ${ }^{\mathrm{b}, \mathrm{d}}$, George A. Stouffer ${ }^{\mathrm{b}, \mathrm{d}}$, Darryl C. \\ Zeldine ${ }^{\mathrm{e}}$, and Craig R. Lee ${ }^{\mathrm{a}, \mathrm{d},{ }^{*}}$ \\ aDivision of Pharmacotherapy and Experimental Therapeutics, Eshelman School of Pharmacy, \\ University of North Carolina, Chapel Hill, NC, USA \\ bDivision of Cardiology, School of Medicine, Department of Biostatistics, University of North \\ Carolina, Chapel Hill, NC, USA \\ 'Gillings School of Global Public Health, University of North Carolina, Chapel Hill, NC, USA \\ ${ }^{\mathrm{d}}$ McAllister Heart Institute, University of North Carolina, Chapel Hill, NC, USA \\ eDivision of Intramural Research, National Institute for Environmental Health Sciences, National \\ Institutes of Health, Research Triangle Park, NC, USA
}

\begin{abstract}
Objective-Accumulating preclinical and epidemiologic evidence has emerged to suggest that modulation of cytochrome P450 (CYP)-mediated eicosanoid metabolism may be a viable vascular protective therapeutic strategy for the secondary prevention of coronary artery disease (CAD). The functional relationship between CYP-derived eicosanoid metabolite levels and vascular dysfunction in humans with established CAD, however, has not been evaluated. Therefore, we characterized the relationship between inter-individual variation in soluble epoxide hydrolase (sEH) and CYP $\omega$-hydroxylase metabolism and established vascular function phenotypes predictive of prognosis in a cohort of patients with atherosclerotic cardiovascular disease.
\end{abstract}

Methods-Plasma epoxyeicosatrienoic acid (EET), dihydroxyeicosatrienoic acid (DHET), and 20-hydroxyeicosatetraenoic acid (20-HETE) levels were quantified by HPLC-MS/MS in 106 patients with stable, angiographically-confirmed CAD. Relationships between biomarkers of CYP-mediated eicosanoid metabolism and vascular function phenotypes were evaluated by Pearson's correlation.

Results-A significant inverse association was observed between 20-HETE levels (a biomarker of CYP $\omega$-hydroxylase metabolism) and brachial artery flow-mediated dilation $(r=-0.255, p=$ 0.010). An inverse association was also observed between 14,15-EET:DHET ratios (a biomarker of sEH metabolism) and both monocyte chemoattractant protein-1 levels $(r=-0.252, p=0.009)$ and a consolidated cellular adhesion molecule 'score' reflecting the levels of E-selectin and P-

(C) 2013 Elsevier Ireland Ltd. All rights reserved.

"Corresponding author. 2317 Kerr Hall, CB\# 7569, UNC Eshelman School of Pharmacy, Chapel Hill, NC 27599, USA. Tel.: +1 919 843 7673; fax: +1 919962 0644. craig_lee@unc.edu (C.R. Lee).

Conflicts of interest

Dr. Zeldin is a co-inventor on U.S. Patent No. 6,531,506 B1 (issued March 11, 2003) titled "Inhibition of Epoxide Hydrolases for the Treatment of Hypertension," U.S. Patent No. 6,693,130 B2 (issued February 17, 2004) titled "Inhibition of Epoxide Hydrolases for the Treatment of Hypertension," and U.S. Patent No. 6,916,843 B1 (issued July 12, 2005) titled "Anti-inflammatory Actions of

Cytochrome P450 Epoxygenase-Derived Eicosanoids." No other authors have conflicts of interest to disclose. 
selectin $(r=-0.216, p=0.027)$. No associations with C-reactive protein or epithelial neutrophilactivating protein-78 levels were observed.

Conclusions-Collectively, these findings demonstrate that enhanced CYP $\omega$-hydroxylase and sEH metabolic function are associated with more advanced endothelial dysfunction and vascular inflammation, respectively, in patients with established atherosclerotic cardiovascular disease. These findings lay the foundation for future clinical research in this area.

\section{Keywords}

Soluble epoxide hydrolase; Epoxyeicosatrienoic acid; 20-Hydroxyeicosatetraenoic acid; Inflammation; Endothelial function

\section{Introduction}

Coronary artery disease (CAD) is the leading cause of morbidity and mortality worldwide and despite recent advances, novel therapies are needed to further improve outcomes. Inflammation and impaired arterial vasodilation (endothelial dysfunction) are key drivers of the pathogenesis and progression of CAD in preclinical models and humans [1]. It is wellestablished that elevated circulating biomarkers of systemic (C-reactive protein) [2] and vascular (chemokines, cellular adhesion molecules [CAMs]) [3,4] inflammation and impaired physiologic measures of endothelial function (brachial artery flow-mediated dilation [FMD]) [5] are predictive of poor prognosis in patients with established CAD. Therefore, CAD patients with persistent vascular inflammation and/or endothelial dysfunction, despite treatment with current standards of care, may be candidates for adjunct therapeutic strategies specifically designed to improve vascular function. Identification of the key pathways that regulate these phenotypes in humans, however, is essential to facilitate the development of targeted therapies for these high-risk subsets of the population.

Accumulating preclinical and epidemiologic evidence has emerged to suggest that modulation of cytochrome P450 (CYP)-mediated eicosanoid metabolism may be a viable clinical therapeutic strategy for the management of cardiovascular disease [6,7]. The CYP2J and CYP2C epoxygenases metabolize arachidonic acid to four epoxyeicosatrienoic acid (EET) regioisomers $(5,6-, 8,9-, 11,12-$, and 14,15-EET), which possess potent vasodilatory and anti-inflammatory effects. The EETs, however, are rapidly metabolized to less active dihydroxyeicosatrienoic acids (DHETs) by soluble epoxide hydrolase (sEH). In parallel, CYP4F and CYP4A $\omega$-hydroxylases generate 20-hydroxyeicosatetraenoic acid (20-HETE), which exhibits potent vasoconstrictive and pro-inflammatory effects. Inhibition of sEH mediated EET hydrolysis and CYP $\omega$-hydroxylase mediated 20-HETE biosynthesis each elicit potent protective effects in preclinical models of endothelial dysfunction and hypertension, nuclear factor (NF)- $\mathrm{kB}$ dependent vascular inflammation, atherosclerosis, and myocardial ischemia-reperfusion injury [6,8-13]. In humans, genetic epidemiology studies have shown that functional polymorphisms in CYP epoxygenases (CYP2J2 and CYP2C8), sEH (EPHX2), and CYP $\omega$-hydroxylases (CYP4F2 and CYP4A11) are associated with the development of cardiovascular disease [14-16].

Furthermore, we recently reported that patients with established atherosclerotic cardiovascular disease exhibit higher plasma EET levels and higher 14,15-EET:DHET ratios compared to healthy individuals at low risk for cardiovascular disease [17]. Conversely, no significant differences in 20-HETE levels were observed. Due to the vascular protective effects of EETs in preclinical models, these data suggest that the presence of cardiovascular disease may lead to a compensatory suppression of sEH-mediated EET hydrolysis in humans. Importantly, pharmacologic inhibitors of sEH [18] and CYP $\omega$-hydroxylase metabolism [19] are currently in development. Although these novel therapies are 
hypothesized to improve prognosis in CAD patients by eliciting protective effects in the vasculature via increasing EET and decreasing 20-HETE levels, respectively, it remains unknown whether CAD patients with dysregulated CYP-mediated eicosanoid metabolism exhibit more advanced vascular dysfunction. A more thorough understanding of the role of CYP-derived EETs and 20-HETE in the regulation of vascular function in patients with established CAD offers enormous potential to identify subsets of the population with dysregulated eicosanoid metabolism (i.e., low EET and/or high 20-HETE levels) who may be most likely to derive benefit from these novel therapies in development.

Therefore, the primary objective of this study was to characterize the relationship between inter-individual variation in CYP-mediated eicosanoid metabolism and key vascular function phenotypes in patients with stable atherosclerotic cardiovascular disease, and determine whether the subset of individuals with enhanced SEH and CYP $\omega$-hydroxylase metabolic function exhibit endothelial dysfunction and advanced inflammation.

\section{Methods}

For an expanded description of the methods, please see the Online Supplement.

\subsection{Study population}

A cohort of 106 individuals with established and stable CAD, defined as $250 \%$ stenosis in one or more major epicardial coronary arteries by coronary angiography, were identified in the University of North Carolina (UNC) Cardiac Catheterization Laboratory, as described $[17,20]$. Eligible participants provided written informed consent and returned $65 \pm 35$ days after their catheterization for a single morning study visit and blood sample collection after fasting overnight and withholding their morning medications. All participants were clinically stable and chest pain free at the time of their study visit. The study protocol was approved by the UNC Biomedical Institutional Review Board.

\subsection{Evaluation of endothelial function}

Endothelium-dependent vasodilation was assessed by brachial artery FMD, as previously described [20]. Brachial artery diameter was assessed at baseline and following reactive hyperemia, and FMD was calculated as the peak percent change in diameter from baseline.

\subsection{Quantification of inflammatory biomarkers}

High sensitivity C-reactive protein (hs-CRP) was quantified in fresh serum using the VITROS $^{\circledR} 5600$ Chemistry System (Ortho-Clinical Diagnostics, Inc., Rochester, NY). Plasma CAM (E-selectin and P-selectin), neutrophil chemokine (epithelial neutrophilactivating protein [ENA]-78) and monocyte chemokine (monocyte chemoattractant protein [MCP]-1) concentrations were quantified using Multi-Analyte Profiling Kits (R\&D Systems, Minneapolis, MN).

\subsection{Quantification of plasma eicosanoids}

Cytochrome P450-derived eicosanoid metabolite concentrations were quantified in plasma after solid phase extraction by high-performance liquid chromatography followed by tandem mass spectrometry (HPLC-MS/MS) as previously described [17]. Plasma 20-HETE levels (the bioactive metabolite of the CYP $\omega$-hydroxylase enzymes) were utilized as a biomarker of CYP $\omega$-hydroxylase pathway function [17]. The 14,15-EET:DHET ratio (a sensitive in vivo biomarker of sEH metabolic function) $[8,15]$ and the sum of EETs (the bioactive metabolites of CYP epoxygenase enzymes) were utilized as biomarkers of CYP epoxygenase pathway function [17] (Supplemental Fig. 1). 


\subsection{Statistical analysis}

Data are presented as mean \pm standard deviation or median (interquartile range) unless otherwise indicated. Since a significant correlation was observed between E-selectin and Pselectin $(r=0.599, p<0.001)$, a consolidated 'CAM score' phenotype was calculated in each individual. No significant correlations were observed among the final five biomarkers of vascular function (Supplemental Table 1), indicating these biomarkers represent five distinct phenotypes.

For the primary analysis, associations between circulating biomarkers of CYP-mediated eicosanoid metabolism (20-HETE, 14,15-EET:DHET ratio, sum EETs) and the five phenotypic indices of vascular function (FMD, CAM score, MCP-1, ENA-78, hs-CRP) were evaluated by Pearson's correlation. A secondary analysis was conducted using a model that adjusted for potential demographic (age, race, gender) and clinical (smoking status, diabetes, obesity, multivessel disease, hypertension, renin-angiotensin system inhibitor use, time post-catheterization) confounders [17]. In order to further assess the eicosanoid-vascular function relationships, vascular phenotypes were compared across eicosanoid metabolism tertiles by analysis of variance (ANOVA) and a post-hoc Student Newman- Keuls test. To minimize the impact of the multiple statistical tests required for this analysis, a false discovery rate (FDR) $q$-value was calculated for each comparison in our primary analysis. Only $q$-values for statistically significant findings $(p<0.05)$ are presented. All statistical analyses were performed using SAS Version 9.2 (SAS Institute, Cary, NC).

\section{Results}

\subsection{Study population}

The population characteristics are shown in Table 1 . The majority of patients had advanced CAD, with 66\% exhibiting multi-vessel disease at their index catheterization, and comorbidities including hypertension (81\%), obesity (54\%), and diabetes (24\%). Medication utilization and revascularization rates were consistent with current clinical practice guidelines.

\subsection{CYP w-hydroxylase pathway}

The relationships between inter-individual variation in CYP-derived eicosanoid levels and vascular function are provided in Table 2. Plasma 20-HETE levels were significantly associated with endothelial dysfunction, such that patients with higher levels of 20-HETE had lower FMD (Fig. 1A, $r=-0.255, p=0.010, q=0.048$ ). A significant inverse association was also observed in the adjusted model $(r=-0.223, p=0.033)$. Similarly, a stepwise trend of lower FMD was observed across increasing tertiles of 20-HETE (Fig. 1B, $p$ for trend $=0.080$ ); however, statistically significant differences were not observed between each tertile. Circulating 20-HETE levels were not associated with either nitroglycerin-mediated dilation $(r=-0.068, p=0.495)$ or baseline brachial artery diameter $(r=0.074, p=0.455)$, indicating that the mechanism underlying the association between 20HETE and FMD is endothelium-dependent and is not explained by differences in arterial size.

A positive relationship between 20-HETE levels and the CAM score was also observed (unadjusted: $r=0.191, p=0.051$; adjusted model: $r=0.222, p=0.031$ ); however, the association was not statistically significant in the primary analysis. In contrast, no association was observed between 20-HETE and either hs-CRP or chemokine levels (Table 2). Similar relationships between 20-HETE levels and each vascular function phenotype were observed after adjusting for or stratifying by aspirin, clopidogrel, beta-blocker, or statin use (data not shown). 


\subsection{CYP epoxygenase pathway}

In contrast to the observed relationship between 20-HETE and endothelial function, no association was observed between FMD and either 14,15-EET:DHET ratios or sum EETs (Table 2). Lower 14,15-EET:DHET ratios (indicative of higher sEH metabolic function) were, however, significantly associated with higher circulating levels of MCP-1 (Fig. 2A, $r$ $=-0.252, p=0.009, q=0.048)$. This association was also significant in the adjusted model $(r=-0.260, p=0.011)$, and across tertiles (Fig. 2B). A significant inverse correlation was also observed between MCP-1 levels and both the sum EET:DHET ratio $(r=-0.245, p=$ 0.012 ) and 12,13-epoxyoctadecenoic acid (EpOME):dihydroxyoctadecenoic acid (DHOME) ratio $(r=-0.201, p=0.040)$. In addition, lower 14,15-EET:DHET ratios were associated with higher CAM scores (Fig. 2C, $r=-0.216, p=0.027, q=0.101$ ), such that those in the lowest tertile had a significantly higher CAM score compared to tertiles 2 and 3 (Fig. 2D). The inverse relationship between CAM score and 14,15-EET:DHET ratio, however, was not statistically significant in the adjusted model $(r=-0.190, p=0.065)$. Similar results were observed with the sum EET:DHET ratio and 12,13-EpOME:DHOME ratio (data not shown).

Consistent with the relationship observed with 14,15-EET:DHET ratios, lower sum EET levels were associated with higher MCP-1 levels (Fig. 3A, $r=-0.283, p=0.003, q=0.045$ ). A significant inverse association was also observed in the adjusted model $(r=-0.243, p=$ 0.017). Furthermore, those with sum EET levels in the lowest two tertiles exhibited significantly higher MCP-1 levels compared to those in the highest tertile (Fig. 3B). When analyzed separately, a significant inverse relationship was observed between MCP-1 and both 8,9-EET $(r=-0.244, p=0.012)$ and 14,15-EET $(r=-0.284, p=0.003)$ levels. No association was observed between sum EETs or 14,15-EET:DHET ratio and either hs-CRP or ENA-78 levels (Table 2). Similar relationships between both 14,15-EET:DHET ratio and sum EETs and each vascular function phenotype were observed after adjusting for or stratifying by aspirin, clopidogrel, beta-blocker, or statin use (data not shown).

\section{Discussion}

Preclinical and genetic epidemiologic studies suggest that increasing CYP-derived EETs and/or decreasing 20-HETE levels may have utility as a vascular protective therapeutic strategy in patients with cardiovascular disease. The functional relationship between CYPmediated eicosanoid metabolism and vascular dysfunction in humans with established cardiovascular disease, however, has not been studied to date. This study demonstrated that higher 20-HETE levels were associated with lower brachial artery FMD and higher CAM levels and lower 14,15-EET:DHET ratios were associated with elevated MCP-1 and CAM levels in a population of patients with stable CAD. These findings are consistent with the vascular effects of 20-HETE and EETs in preclinical models, and suggest that enhanced CYP $\omega$-hydroxylase and sEH metabolic function predispose stable CAD patients to more advanced endothelial dysfunction and vascular inflammation, respectively.

It is well-established that biomarkers of vascular function are associated with prognosis in patients with established CAD [1]. For example, persistently elevated circulating levels of hs-CRP, CAMs and MCP-1, or genetic predisposition to higher ENA-78 levels, have each been associated with poorer survival in patients with established CAD [2-4,21]. Similarly, CAD patients with persistently impaired FMD despite optimized therapy have a higher risk of cardiovascular events, suggesting that adjunct therapies that improve FMD may subsequently improve prognosis [5]. Identification of key regulators of these distinct vascular phenotypes predictive of prognosis offers enormous potential to facilitate the development of targeted therapies designed to improve vascular function and prognosis in high-risk subsets of the population. 
The observed inverse association between circulating 20-HETE levels and brachial artery FMD is consistent with preclinical studies in which 20-HETE directly induces reactive oxygen species (ROS) production and endothelial nitric oxide synthase uncoupling, reduces NO availability, and impairs endothelial-dependent vasodilation [9]. An inverse association between urinary excretion of 20-HETE, which is reflective of renal 20-HETE biosynthesis [13], and FMD has previously been reported in humans without known CAD [22]. Our findings with circulating 20-HETE are consistent with this report; however, we are the first to demonstrate an association between 20-HETE and FMD in humans with established CAD. We also observed a positive relationship between plasma 20-HETE levels and circulating CAMs that is consistent with preclinical studies demonstrating that 20-HETE directly induces CAM expression in endothelial cells through activation of NF- $\kappa B$ [23]. In our previous case-control analysis, we did not observe a significant difference in 20-HETE levels in stable CAD patients compared to healthy individuals; however, CAD patients who were not treated with a renin-angiotensin system inhibitor exhibited modestly higher 20HETE levels compared to healthy individuals [17]. Our current findings suggest that despite being treated with current standard of care therapies (including renin-angiotensin system inhibitors), CAD patients with the highest 20-HETE levels may be predisposed to more advanced endothelial dysfunction and vascular inflammation, and therapeutic strategies that directly decrease 20-HETE biosynthesis may improve vascular function in this high-risk population.

Consistent with a series of preclinical studies and our hypothesis, we are also the first to report that lower EET:DHET ratios, a biomarker of enhanced sEH metabolic function, were associated with higher plasma MCP-1 and CAM levels. Indeed, EETs exert potent antiinflammatory effects in the vasculature in preclinical models via attenuation of NF- $\mathrm{kB}$ activation and chemokine and CAM expression [10,11], and these effects are potentiated via inhibition of sEH [6]. These data are consistent with a recent report demonstrating that CYP2J2 and CYP2C8 are expressed in human monocytes, and CYP epoxygenase metabolic function attenuates monocyte/macrophage activation [24]. In addition, a recent study in mice demonstrated that sEH activity in bone marrow-derived cells, the primary site of chemokine biosynthesis, is a key regulator of plasma epoxide:diol ratios [25]. We previously reported that plasma epoxide:diol ratios were significantly higher in patients with established CAD compared to healthy individuals at low risk for cardiovascular disease [17]. Due to the aforementioned vascular protective effects of EETs, these data suggest that the presence of cardiovascular disease may lead to a compensatory suppression of sEH metabolic function and higher EET levels in humans. However, our current findings suggest that, in spite of a potential compensatory increase in EET levels overall, the subset of CAD patients with the highest sEH metabolic function may be predisposed to more advanced vascular inflammation. Consequently, therapeutic strategies that further increase EET levels, most notably sEH inhibitors, may represent an effective secondary prevention strategy by attenuating vascular inflammation and improving prognosis in this high-risk population.

In contrast, we did not observe an association between 14,15-EET:DHET ratio and FMD which is surprising given the known vasodilatory properties of EETs in preclinical models and our previous finding that $E P H X 2$ genotype is associated with vasodilator responses in healthy individuals [26]. In addition to the different populations, it should be noted that brachial artery FMD (an index of NO bioavailability and conduit arterial function) was evaluated in the current study while bradkyinin-induced changes in forearm blood flow (a phenotype more reflective of microvascular function) was evaluated in the previous study. Although these contrasting results may not be surprising given the more prominent role of EETs in the regulation of microvascular compared to macrovascular tone in preclinical models [7], it is important to note that our current findings are consistent with previous studies demonstrating that CYP epoxygenase inhibition does not impair endothelium- 
dependent vasodilator responses in CAD patients in vivo [27], or in isolated coronary arterioles from patients undergoing cardiac surgery [28], due to the presence of enhanced oxidative stress. Collectively, these data suggest that the vasodilatory effects of CYPderived EETs may be overwhelmed and masked by the presence of ROS in populations with underlying vascular inflammation and remodeling, such as atherosclerotic cardiovascular disease. This hypothesis requires rigorous investigation in prospective interventional studies.

No associations were observed between biomarkers of CYP-mediated eicosanoid metabolism and hs-CRP. While chemokines and CAMs mediate inflammation in the vasculature, hs-CRP is an acute-phase reactant released from the liver indicative of systemic inflammation [29]. Consequently, our findings indicate that CYP-derived eicosanoids may be important in the regulation of vascular, but not hepatic or systemic, inflammation in humans. This is consistent with previous observations in preclinical models demonstrating that the hepatic inflammatory response to endotoxin is not attenuated in Ephx 2 knockout mice [30]. Furthermore, no relationship between CYP-mediated eicosanoid metabolism and the neutrophil chemokine ENA-78 was observed. We have reported that genetic potentiation of the CYP epoxygenase pathway attenuates induction of ENA-78 expression and neutrophil infiltration in mice [11]. In contrast, CYP epoxygenase enzymes are not expressed in human polymorphonuclear cells [24], suggesting the presence of species differences in the contribution of CYP-derived eicosanoids to the regulation of neutrophil activation.

Our analysis has limitations that must be acknowledged. First, although the observed associations were consistent with prior preclinical studies, the cross-sectional design does not allow us to establish a cause-and-effect relationship between eicosanoid metabolism and vascular function. Second, our analysis included multiple statistical comparisons and was limited by its relatively small sample size. To account for the possibility of false-positive findings, we calculated an FDR $q$-value for each comparison. All statistically significant associations had an FDR of $10 \%$ or less, and were in directions consistent with prior preclinical evidence, giving us a higher level of confidence in our results. Furthermore, at $a$ $=0.05$, there was greater than $80 \%$ power to detect an $r^{2}$ of 0.07 for each eicosanoid metabolism-vascular phenotype relationship, which is similar in magnitude to the wellestablished and clinically relevant association observed between body mass index and hsCRP in previous studies [29]. This demonstrates that we had ample statistical power to detect biologically meaningful and clinically relevant relationships in the current study. Importantly, validation of the observed relationships in an independent cohort will ultimately be necessary. Lastly, the population under investigation had established atherosclerotic cardiovascular disease and thus was being treated according to current clinical practice guidelines, including multiple medications that impact vascular function (i.e., statins, low-dose aspirin, clopidogrel, beta-blockers). Moreover, the impact of these medications (most notably aspirin which modifies prostanoid levels) on CYP-mediated eicosanoid metabolism has not been rigorously evaluated in preclinical models or humans, and thus remains unknown. Therefore, we cannot elucidate whether the observed relationships were modified by medication use. Although our adjusted and stratified analyses showed similar associations between biomarkers of CYP-mediated eicosanoid metabolism and vascular function phenotypes irrespective of medication use, evaluation of the direct impact of medication use on EET and 20-HETE levels, as well as the modifying effect of medication use on the observed eicosanoid-vascular function relationships, is beyond the scope of the current investigation and requires further study. Importantly, since pharmacologic agents in development that increase EETs (sEH inhibitors) or decrease 20HETE (CYP $\omega$-hydroxylase inhibitors) would most likely be used as a secondary prevention strategy for $\mathrm{CAD}$, in addition to these standard of care medications, our study was designed to specifically characterize associations between biomarkers of CYP-mediated eicosanoid metabolism and vascular function in a population of stable, appropriately treated patients 
with established CAD. These data lay a foundation for the rational design of subsequent studies that aim to directly evaluate the effects of novel therapies that decrease 20-HETE and increase EET levels on vascular function in patients with established CAD.

\title{
5. Conclusions
}

This cross-sectional analysis demonstrates that higher circulating 20-HETE levels are associated with lower brachial artery FMD and higher CAMs and lower 14,15-EET:DHET ratios are associated with elevated MCP-1 and CAM levels in a population of patients with stable CAD. These findings suggest that enhanced CYP $\omega$-hydroxylase and sEH metabolic function may predispose patients with established atherosclerotic cardiovascular disease to more advanced endothelial dysfunction and vascular inflammation despite current standards of care. These findings lay a critical foundation for future clinical research in this area, including the rational design of interventional proof-of-concept studies that seek to define the vascular protective effects and safety of decreasing CYP-mediated 20-HETE biosynthesis and/or decreasing sEH-mediated EET hydrolysis in patients with established CAD.

\section{Supplementary Material}

Refer to Web version on PubMed Central for supplementary material.

\section{Acknowledgments}

\begin{abstract}
Funding
This publication was made possible by a predoctoral training program in Integrative Vascular Biology supported by the NIH/NHLBI (T32 HL069768) and a predoctoral fellowship from the American Heart Association (11PRE7240059) to Dr. Schuck, a pre-doctoral fellowship from the American Foundation for Pharmaceutical Education to Dr. Theken, a Beginning Grant-in-Aid from the American Heart Association and a pilot grant from the North Carolina Translational and Clinical Sciences Institute to Dr. Lee, funds from the Intramural Research Program of the NIH/NIEHS to Dr. Tomer (Z01 ES050167) and Dr. Zeldin (Z01 ES025034), and by grant KL2RR025746 from the NIH/NCRR and NIH/NCATS. Its contents are solely the responsibility of the authors and do not necessarily represent the official views of the NIH.
\end{abstract}

The authors gratefully acknowledge the UNC Clinical and Translational Research Center staff for their assistance, and the UNC Cytokine Analysis facility for performing the Bioplex assays.

\section{References}

1. Hansson GK. Inflammation, atherosclerosis, and coronary artery disease. N Engl J Med. 2005; 352:1685-95. [PubMed: 15843671]

2. Ridker PM, Cannon CP, Morrow D, et al. C-reactive protein levels and outcomes after statin therapy. N Engl J Med. 2005; 352:20-8. [PubMed: 15635109]

3. de Lemos JA, Morrow DA, Blazing MA, et al. Serial measurement of monocyte chemoattractant protein-1 after acute coronary syndromes: results from the A to Z trial. J Am Coll Cardiol. 2007; 50:2117-24. [PubMed: 18036447]

4. Blankenberg S, Rupprecht HJ, Bickel C, et al. Circulating cell adhesion molecules and death in patients with coronary artery disease. Circulation. 2001; 104:1336-42. [PubMed: 11560847]

5. Kitta Y, Obata JE, Nakamura T, et al. Persistent impairment of endothelial vasomotor function has a negative impact on outcome in patients with coronary artery disease. J Am Coll Cardiol. 2009; 53:323-30. [PubMed: 19161880]

6. Deng Y, Theken KN, Lee CR. Cytochrome P450 epoxygenases, soluble epoxide hydrolase, and the regulation of cardiovascular inflammation. J Mol Cell Cardiol. 2010; 48:331-41. [PubMed: 19891972] 
7. Roman RJ. P-450 metabolites of arachidonic acid in the control of cardiovascular function. Physiol Rev. 2002; 82:131-85. [PubMed: 11773611]

8. Seubert JM, Sinal CJ, Graves J, et al. Role of soluble epoxide hydrolase in postischemic recovery of heart contractile function. Circ Res. 2006; 99:442-50. [PubMed: 16857962]

9. Cheng J, Wu CC, Gotlinger KH, et al. 20-Hydroxy-5,8,11,14-eicosatetraenoic acid mediates endothelial dysfunction via IkappaB kinase-dependent endothelial nitric-oxide synthase uncoupling. J Pharmacol Exp Ther. 2010; 332:57-65. [PubMed: 19841472]

10. Node K, Huo Y, Ruan X, et al. Anti-inflammatory properties of cytochrome P450 epoxygenasederived eicosanoids. Science. 1999; 285:1276-9. [PubMed: 10455056]

11. Deng Y, Edin ML, Theken KN, et al. Endothelial CYP epoxygenase over-expression and soluble epoxide hydrolase disruption attenuate acute vascular inflammatory responses in mice. FASEB J. 2011; 25:703-13. [PubMed: 21059750]

12. Zhang LN, Vincelette J, Cheng Y, et al. Inhibition of soluble epoxide hydrolase attenuated atherosclerosis, abdominal aortic aneurysm formation, and dyslipidemia. Arterioscler Thromb Vasc Biol. 2009; 29:1265-70. [PubMed: 19667112]

13. Liu X, Zhao Y, Wang L, et al. Overexpression of cytochrome P450 4F2 in mice increases 20hydroxyeicosatetraenoic acid production and arterial blood pressure. Kidney Int. 2009; 75:128896. [PubMed: 19279555]

14. Spiecker M, Darius H, Hankeln T, et al. Risk of coronary artery disease associated with polymorphism of the cytochrome P450 epoxygenase CYP2J2. Circulation. 2004; 110:2132-6. [PubMed: 15466638]

15. Lee CR, North KE, Bray MS, et al. Genetic variation in soluble epoxide hydrolase (EPHX2) and risk of coronary heart disease: the Atherosclerosis Risk in Communities (ARIC) study. Hum Mol Genet. 2006; 15:1640-9. [PubMed: 16595607]

16. Ward NC, Tsai IJ, Barden A, et al. A single nucleotide polymorphism in the CYP4F2 but not CYP4A11 gene is associated with increased 20-HETE excretion and blood pressure. Hypertension. 2008; 51:1393-8. [PubMed: 18391101]

17. Theken KN, Schuck RN, Edin ML, et al. Evaluation of cytochrome P450-derived eicosanoids in humans with stable atherosclerotic cardiovascular disease. Atherosclerosis. 2012; 222:530-6. [PubMed: 22503544]

18. Chen D, Whitcomb R, MacIntyre E, et al. Pharmacokinetics and pharmaco-dynamics of AR9281, an inhibitor of soluble epoxide hydrolase, in single- and multiple-dose studies in healthy human subjects. J Clin Pharmacol. 2012; 52:319-28. [PubMed: 21422238]

19. Williams JM, Murphy S, Burke M, Roman RJ. 20-hydroxyeicosatetraeonic acid: a new target for the treatment of hypertension. J Cardiovasc Pharmacol. 2010; 56:336-44. [PubMed: 20930591]

20. Lee CR, Bass A, Ellis K, et al. Relation between digital peripheral arterial tonometry and brachial artery ultrasound measures of vascular function in patients with coronary artery disease and in healthy volunteers. Am J Cardiol. 2012; 109:651-7. [PubMed: 22154090]

21. Zineh I, Beitelshees AL, Welder GJ, et al. Epithelial neutrophil-activating peptide (ENA-78), acute coronary syndrome prognosis, and modulatory effect of statins. PLoS One. 2008; 3:e3117. [PubMed: 18769620]

22. Ward NC, Rivera J, Hodgson J, et al. Urinary 20-hydroxyeicosatetraenoic acid is associated with endothelial dysfunction in humans. Circulation. 2004; 110:438-43. [PubMed: 15262846]

23. Ishizuka T, Cheng J, Singh H, et al. 20-Hydroxyeicosatetraenoic acid stimulates nuclear factorkappaB activation and the production of inflammatory cytokines in human endothelial cells. J Pharmacol Exp Ther. 2008; 324:103-10. [PubMed: 17947496]

24. Bystrom J, Wray JA, Sugden MC, et al. Endogenous epoxygenases are modulators of monocyte/ macrophage activity. PLoS One. 2011; 6:e26591. [PubMed: 22028915]

25. Fromel T, Jungblut B, Hu J, et al. Soluble epoxide hydrolase regulates hematopoietic progenitor cell function via generation of fatty acid diols. Proc Natl Acad Sci U S A. 2012; 109:9995-10000. [PubMed: 22665795]

26. Lee CR, Pretorius M, Schuck RN, et al. Genetic variation in soluble epoxide hydrolase (EPHX2) is associated with forearm vasodilator responses in humans. Hypertension. 2011; 57:116-22.

[PubMed: 21098312] 
27. Fichtlscherer S, Dimmeler S, Breuer S, Busse R, Zeiher AM, Fleming I. Inhibition of cytochrome P450 2C9 improves endothelium-dependent, nitric oxide-mediated vasodilatation in patients with coronary artery disease. Circulation. 2004; 109:178-83. [PubMed: 14662709]

28. Larsen BT, Gutterman DD, Sato A, et al. Hydrogen peroxide inhibits cytochrome p450 epoxygenases: interaction between two endothelium-derived hyperpolarizing factors. Circ Res. 2008; 102:59-67. [PubMed: 17975109]

29. Tracy RP, Psaty BM, Macy E, et al. Lifetime smoking exposure affects the association of Creactive protein with cardiovascular disease risk factors and subclinical disease in healthy elderly subjects. Arterioscler Thromb Vasc Biol. 1997; 17:2167-76. [PubMed: 9351386]

30. Fife KL, Liu Y, Schmelzer KR, et al. Inhibition of soluble epoxide hydrolase does not protect against endotoxin-mediated hepatic inflammation. J Pharmacol Exp Ther. 2008; 327:707-15. [PubMed: 18815352]

\section{Appendix A. Supplementary data}

Supplementary data related to this article can be found at http://dx.doi.org/10.1016/ j.atherosclerosis.2013.01.034. 

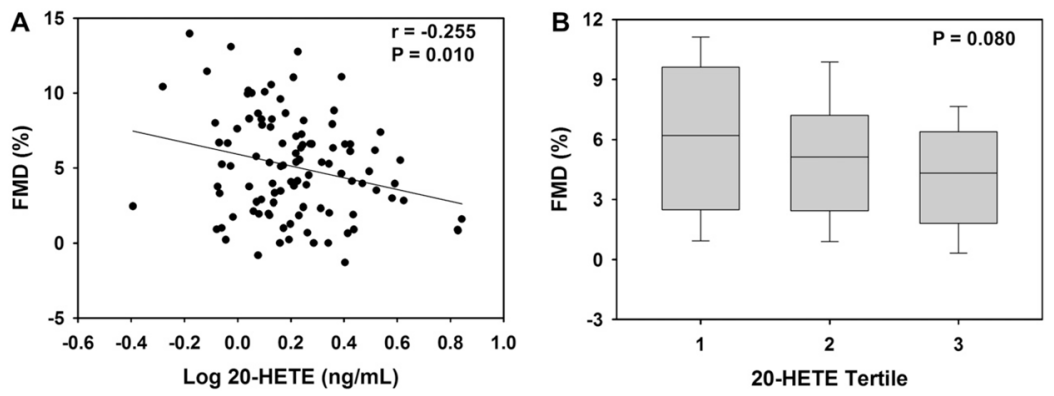

Fig. 1.

Association between 20-HETE levels and FMD. (A) The correlation between 20-HETE and FMD is displayed. (B) The distribution of FMD across 20-HETE tertiles is displayed using box plots (tertile 1: $0.40-1.29 \mathrm{ng} / \mathrm{mL}$, tertile $2: 1.30-1.85 \mathrm{ng} / \mathrm{mL}$, tertile $3: 1.86-6.97 \mathrm{ng} /$ $\mathrm{mL}$ ). The horizontal line within the box represents the median, the edges of the box represent the 25 th and 75 th percentiles, and the error bars represent the 10th and 90th percentiles. The ANOVA $p$-value for the trend is provided. 



Fig. 2.

Association between 14,15-EET:DHET ratios and biomarkers of vascular inflammation. The correlation between 14,15-EET:DHET ratio and (A) MCP-1 and (C) CAM score is displayed. The distribution of (B) MCP-1 and (D) CAM score across 14,15-EET:DHET ratio tertiles is displayed using box plots (tertile 1: $0.040-0189 \mathrm{ng} / \mathrm{mL}$, tertile 2: $0.190-0.541$ $\mathrm{ng} / \mathrm{mL}$, tertile $3: 0.542-1.82 \mathrm{ng} / \mathrm{mL}$ ). The ANOVA $p$-value for the trend is provided. $* p<$ 0.05 versus tertile $3 .{ }^{\dagger} p<0.05$ versus tertile 2 . 

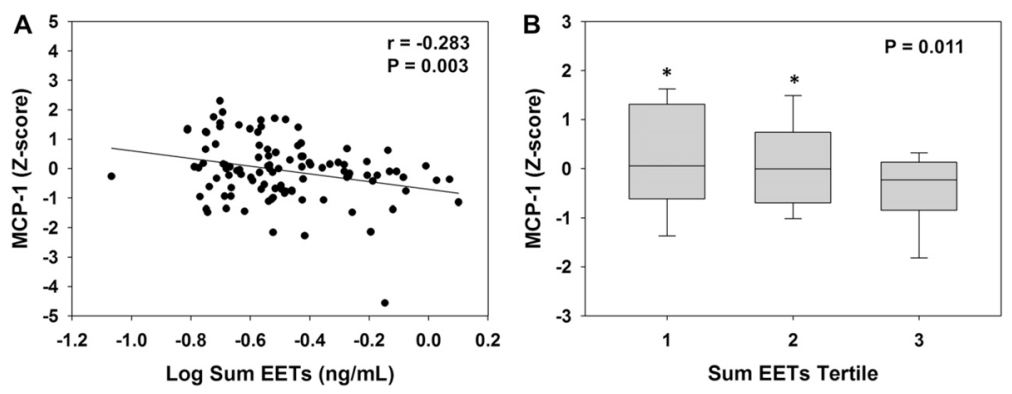

Fig. 3.

Association between sum EETs and MCP-1 levels. (A) The correlation between sum EETs and MCP-1 is displayed. (B) The distribution of MCP-1 across sum EETs tertiles is displayed using box plots (tertile 1: $0.086-0.251 \mathrm{ng} / \mathrm{mL}$, tertile $2: 0.252-0.375 \mathrm{ng} / \mathrm{mL}$, tertile $30.376-1.26 \mathrm{ng} / \mathrm{mL})$. The ANOVA $p$-value for the trend is provided. $* p<0.05$ versus tertile 3 . 


\section{Table 1}

Study population characteristics.

\begin{tabular}{ll}
\hline Characteristic & \\
\hline $\mathrm{N}$ & 106 \\
Age (years) & $58 \pm 10$ \\
Female (\%) & $35(33 \%)$ \\
African-American (\%) & $18(17 \%)$ \\
Body mass index (kg/m²) & $30 \pm 6$ \\
Obese (BMI $\left.230 \mathrm{~kg} / \mathrm{m}^{2} ; \%\right)$ & $57(54 \%)$ \\
Current smoker (\%) & $23(22 \%)$ \\
Diabetes (\%) & $25(24 \%)$ \\
Hypertension (\%) & $86(81 \%)$ \\
Previous myocardial infarction & $39(37 \%)$ \\
Multivessel disease & $70(66 \%)$ \\
Recent revascularization procedure & $70(66 \%)$ \\
Systolic blood pressure (mmHg) & $136 \pm 17$ \\
Diastolic blood pressure (mmHg) & $80 \pm 10$ \\
Total cholesterol (mmol/L) & $4.04(1.32)$ \\
LDL cholesterol (mmol/L) & $2.20(0.96)$ \\
HDL cholesterol (mmol/L) & $1.22(0.41)$ \\
Triglycerides (mmol/L) & $1.11(0.84)$ \\
ACE inhibitor or ARB (\%) & $66(62 \%)$ \\
Beta-blocker (\%) & $89(84 \%)$ \\
Statin (\%) & $99(93 \%)$ \\
Aspirin (\%) & $103(97 \%)$ \\
Clopidogrel (\%) & $83(78 \%)$ \\
\hline & \\
&
\end{tabular}

Data presented as mean \pm standard deviation, median (interquartile range) or count (proportion).

$\mathrm{ACE}=$ angiotensin-converting enzyme, $\mathrm{ARB}=$ angiotensin receptor blocker, $\mathrm{HDL}=$ high density lipoprotein, $\mathrm{LDL}=$ low density lipoprotein.

$a_{59 / 106}$ underwent a percutaneous coronary intervention and 11/106 underwent a coronary artery bypass grafting procedure between screening and the study visit. 
